\title{
System of indication of directions and warnings against collisions for locomotion of people with impaired vision in structured environments
}

\author{
Álvaro José Belota de Oliveira Saraiva e Walter Andrés Vermehren Valenzuela \\ ${ }^{1}$ Universidade do Estado do Amazonas - UEA, Discente do Curso de Eng. de Controle e Automação, Manaus, Amazonas, \\ Brasil. E-mail: alvarobelota.s@gmail.com. \\ ${ }^{1}$ Universidade do Estado do Amazonas - UEA, Coordenador do Curso de Eng. de Controle e Automação, Manaus, Amazonas, \\ Brasil, Eng. Indl Mecânico, Doutor em Instrumentação e Controle - UFCG. E-mail: wvalenzuela7@ gmail.com.
}

\begin{abstract}
This article deals with the development of Assistive Technologies in order to provide or expand functional abilities of people with visual impairments, analyzing in general is perceived that the technological evolution goes toward making life easier, this project is exactly the creation an indicator of direction and warning against collisions based on a data processing unit, approximate location system received signal strength indicator (RSSI) or indicator intensity of the residual signal in Portuguese, in structured environments coupled with a recognition unit obstacles that allows guide people with impaired vision by sending beeps.
\end{abstract}

Key-words: System Approximate Location RSSI, Structured environments, Obstacle recognition.

\section{Sistema de indicação de direções e alerta contra colisões para locomoção de pessoas com deficiência visual em ambientes estruturados}

\begin{abstract}
RESUMO
Este artigo trata do desenvolvimento de Tecnologias Assistivas com intuito de proporcionar ou ampliar habilidades funcionais de pessoas com deficiência visual, analisando de uma maneira geral percebe-se que a evolução tecnológica caminha na direção de tornar a vida mais fácil, este projeto trata exatamente da criação de um indicador de direção e alerta contra colisões tendo como base uma unidade de processamento de dados, sistema de localização aproximada received signal strength indicator (RSSI), ou Indicador de intensidade do sinal residual em português, em ambientes estruturados aliado a uma unidade de reconhecimento de obstáculos que possibilite orientar o deficiente por meio do envio de sinais sonoros.
\end{abstract}

Palavras-chaves: Sistema de Localização Aproximada RSSI, Ambientes estruturados, Reconhecimento de Obstáculos.

\section{INTRODUÇÃO}

No Brasil segundo o IBGE (2010) estima-se que existam mais de 35 milhões de pessoas com algum tipo de deficiência visual, por esse motivo o desenvolvimento de tecnologias assistivas é necessário para o auxilio dessas pessoas [1].

A acessibilidade trata da possibilidade e condição de alcance para utilização, com segurança e autonomia, dos ambientes estruturados, no entanto as dificuldades encontradas por pessoas com deficiência visual retratam possibilidades mínimas de locomoção tanto pela dificuldade de senso de direção ao ponto onde se pretende chegar quanto pelos obstáculos físicos encontrados pelo caminho.

Este artigo propõe a criação de um sistema de indicação de direção e alerta contra colisões para pessoas com deficiência, por meio do envio de sinais sonoros executados por meio de um módulo de áudio gerenciado por uma unidade central que será responsável também pela

ITEGAM - JETIA Vol. xx, No. x. Mês de ano. Manaus - Amazonas, Brasil. ISSN 2447-0228 (ONLINE). 
Álvaro José Belota de Oliveira Saraiva \& Walter Andrés Vermehren Valenzuela/ITEGAM-JETIA Vol. 01, No 04, pp.35-39. Dezembro, 2015.

aquisição e tratamento de dados de um sistema de localização aproximada criado por meio da distribuição de uma rede ZigBee baseado em propriedades geométricas utilizando a técnica de RSSI em um ambiente estruturado interligado a um sistema de deteccão de obstáculos feito com a utilização de sensores de obstáculos.

Para projetar um sistema embarcado que por meio de uma unidade de controle consiga processar informações recebidas, via ZigBee, de um sistema de localização aproximada baseado na técnica de RSSI e pela leitura de sensores responsáveis pela detecção de obstáculos para auxiliar pessoas com deficiência visual quanto a direção a ser tomada e contra a colisão contra possíveis obstáculos que possam encontrar em um ambiente estruturado.

\section{DESCRIÇÃO DE MATERIAIS E MÉTODOS}

Para desenvolvimento deste projeto, são utilizados uma comunicação sem fio ZigBee; sensores ultrassônicos HC-SR04 e microcontrolador Arduino Uno. Além desses materiais também é necessária a utilização de algumas ferramentas de software que serão apresentadas no decorrer do artigo.

\section{II.1ESTRATÉGIA DE ESTIMATIVA DE LOCALIZAÇÃO}

De acordo com [2] o RSSI é um indicador de intensidade de sinal de rádio onde é possível estabelecer, em $\mathrm{dBm}$, uma medida de intensidade do sinal recebido por um receptor de maneira facilitada por sua simplicidade de medição e por não necessitar de hardware adicional para a medida da intensidade do sinal. Sendo assim sua utilização é um método para estimar a distância entre emissor e receptor em uma rede estabelecida em ambiente indoor.

De acordo com [3] a fórmula necessária para o cálculo da RSSI é dada por:

$$
R S S I=P_{0}+10_{n} \log \left(\frac{d^{\prime}}{d^{\prime} 0}\right)+X
$$

onde: RSSI é a potência recebida do sinal de rádio (em $\mathrm{dbm}), \mathrm{P}_{0}$ é a potência recebida na medida de referência, $10_{n}$ é o resultado do cálculo do path loss da variável de influência do ambiente no sinal, d é a distância que o sinal percorreu, $d_{0}$ é a distância de referência do modelo e $X$ é a variável que representa a influência do ambiente no sinal.
Conhecendo o valor de RSSI, em $\mathrm{dBm}$, posteriormente o isolamento da variável d possibilita o cálculo da distancia para estimar a localização da pessoa com deficiência no ambiente.

\section{2 TECNOLOGIA ZIGBEE}

Segundo [4] a especificação ZigBee foca nos protocolos de alto nível para dispositivos pequenos de rádio digital e com baixo consumo de energia, o que o torna tão atrativo para utilização em projetos que requeiram o mínimo de consumo de energia possível. Trata-se de uma tecnologia relativamente simples, que utiliza um protocolo de pacotes de dados com características específicas, sendo projetado para oferecer flexibilidade quanto aos tipos de dispositivos que pode controlar.

Os módulos Xbee permitem a comunicação numa rede ZigBee, possibilitando que diversos dispositivos estejam conectados uns com os outros tornando possível a utilização da técnica do RSSI e por esse motivo será responsável pela localização aproximada de pessoas com deficiência visual baseado em fórmulas matemáticas e da intensidade do sinal enviado. Sua utilização se torna facilitada quando acoplados aos kits Arduino que permite uma interação com suas portas de entrada e saída podendo ser promover a comunicação entre dois ou mais Arduínos.

Desenvolvido pela Digi para configuração dos módulos, atualização de firmwares e outras funções, o software utilizado será o XCTU, que possibilita fazer a configuração dos módulos de maneira simples e intuitiva.

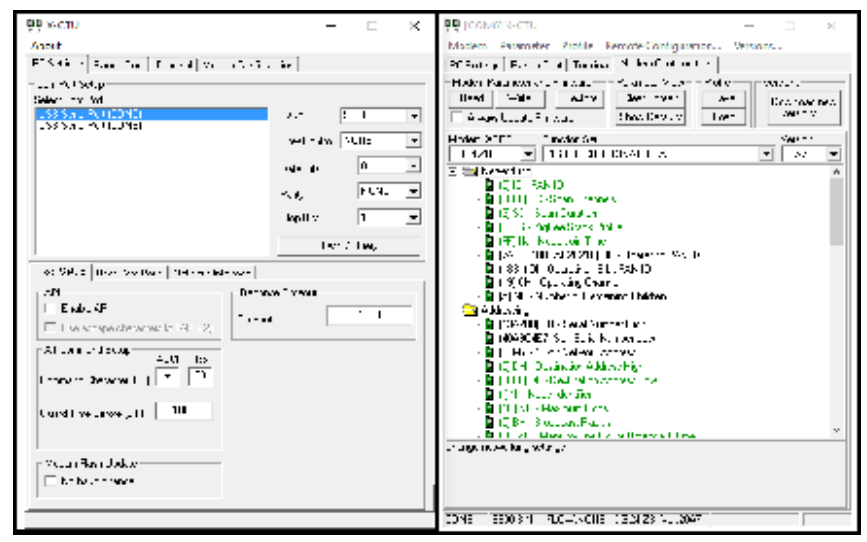

Figura 1. Software X-CTU.

\section{II.3 IDENTIFICAÇÃO DE OBSTÁCULOS}

Para a identificação de possíveis obstáculos foram utilizados sensores ultrassônicos, que tem seu uso muito difundido devido ao seu baixo custo e grande disponibilidade no mercado aliado a sua capacidade de obter a distância a obstáculos de forma relativamente simples e precisa, e a imunidade a alguns agentes externos, como iluminação [5]. 
Conforme [6], "Com um sensor ultrassônico pode-se obter a informação de distância a partir da técnica de tempo de vôo. Mede-se o tempo entre a emissão do pulso e o retorno do eco". Por conseguir precisar a distância até o objeto o sensor HCSR04 se torna uma solução para a utilização no projeto, haja vista que sua aplicação é totalmente compatível com o arduíno.

O cálculo da distância pode ser feito de acordo com o tempo em que o pino ECHO permanece em nível alto após o pino Trigger ter sido colocado em nível alto.

$$
D=\frac{\text { Tempoechoo }{ }^{*} \text { Velocidade do som }}{2}
$$

Considerando-se um range para a distância que varia de 0,2 $\mathrm{cm}$ e $400 \mathrm{~cm}$ e uma velocidade de som aproximada de $340 \mathrm{~m} / \mathrm{s}$ [7].

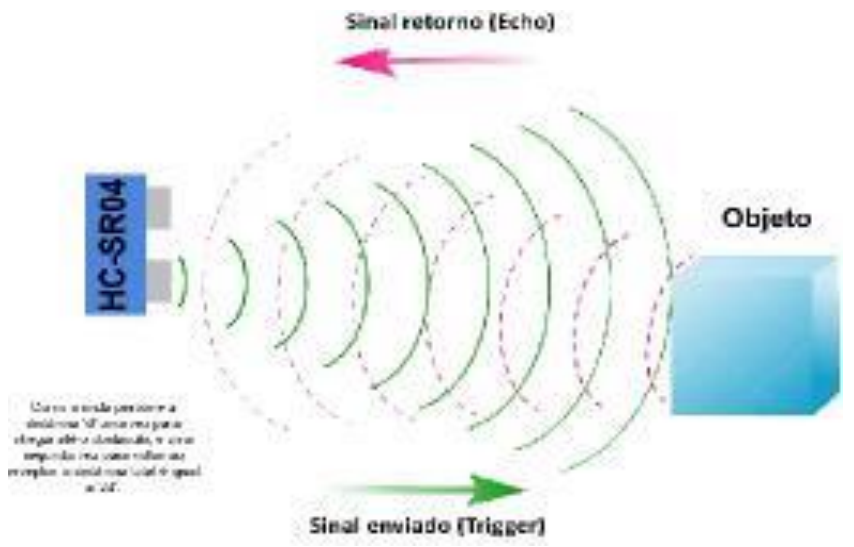

Figura 2. Esquema de Funcionamento do Sensor Ultrassônico. Fonte.http://blog.filipeflop.com/wpcontent/uploads/2015/07/HC_SR04_Trigger_E cho.jpg, adaptado pelo Autor.

\section{II.4 MICROCONTROLADOR ARDUINO E PROGRAMAÇÃO}

O Arduino é um microcontolador, que possui um processador Atmega (Atmel) que pode ser programado por meio do software IDE Arduino 1.0.6® disponibilizado pelo fabricante. Como características do Arduino Uno pode-se citar: uma tensão de operação de $5 \mathrm{~V}$, a tensão de entrada de 7 a 12V, 14pinos de entrada digitais e 6 de entradas analógicas, 6 das entradas digitais são ajustáveis em PWM.

O arduino será responsável pelo controle do sistema, recebendo e processando informações dos sensores e ZigBee enviando a seguir através do módulo de áudio indicações quanto a localização de pontos definidos do ambiente estruturado que sejam importantes para que a pessoa com deficiência consiga ter referências para uma lozalização aproximada eficaz, a figura 6 mostra de forma simples como se dá o funcionamento do sistema de controle [8].

\section{II.5 MÓdULO DE ÁUdiO}

Para o envio de informações de áudio a pessoa com deficiência visual foi utilizado o módulo WTV020M01 que possui slote para cartão de memória que dá suporte para que os arquivos salvos possam ser reproduzidos em formato de áudio Mp3 com o auxílio de um fone, sua utilização é possível em conjunto com o arduíno que através de programação permite que apenas áudios necessários para cada situação, em que a pessoa com deficiência visual esteja, sejam reproduzidos [9].

\section{6 ESTRUTURA}

Para efeito de testes é necessário a criação de um protótipo composto por um sistema de detecção de obstáculos com o intuito de testar a eficiência com que o sensor escolhido se comporta, um sistema de localização aproximada baseado em uma rede RSSI e do módulo de áudio. Os sistemas foram implementados individualmente para a execução dos testes e futuramente serão conectados por meio de uma estrutura física que será portada pela pessoa com deficiência visual.

O sistema foi desenvolvido com o auxílio de softwares para a programação do Arduino tanto para o sistema de detecção de obstáculos quanto para a utilização com o ZigBee que teve sua configuração executada por meio do software X-CTU.

\section{RESULTADOS E DISCUSSÕES}

\section{III.1 ESTIMATIVA DE LOCALIZAÇÃO}

Para efeito de testes foram utilizados módulos Xbee da série 2 que conta com pilha protocolar robusta para rede onde os dispositivos podem rotear informações diretamente uns para os outros, sendo definidos como dispositivo coordenador que atuam como um gerente e dispositivo Roteador que troca dados com outros dispositivos. Utilizando os pinos RSSI de cada dispositivo foi possível extrair um sinal representando o valor de sua intensidade em PulseWidth Modulation (PWM), ou modulação por largura de pulso em português, uma técnica que consiste em manter a frequência de uma onda quadrada fixa e variar o tempo em que o sinal fica em nível lógico alto, para a obtenção desses valores em $\mathrm{dBm}$ foi necessário que o sinal fosse transformado em um número inteiro, que foi possível utilizando a biblioteca XBee.h por meio da programação no software IDE Arduino. Levando em conta que a configuração do Xbee por meio do software XCTU permitiu que cada dispositivo emitisse um pacote de dados específico, uma espécie de identidade para cada dispositivo, foi possível fazer a leitura da distância entre os módulos Xbee podendo diferenciá-los.

A partir disso com o uso da fórmula necessária para o cálculo da RSSI como demonstrado anteriormente foi possível mensurar a distância entre os dispositivos sendo demonstrada por meio do monitor serial do software do Arduino como pode ser observado na figura abaixo, para a comunicação entre vários dispositivos o envio de dados por pacote foi de suma importância para que a programação possa ser feita de maneira a levar a diferenciação entre os dispositivos. 


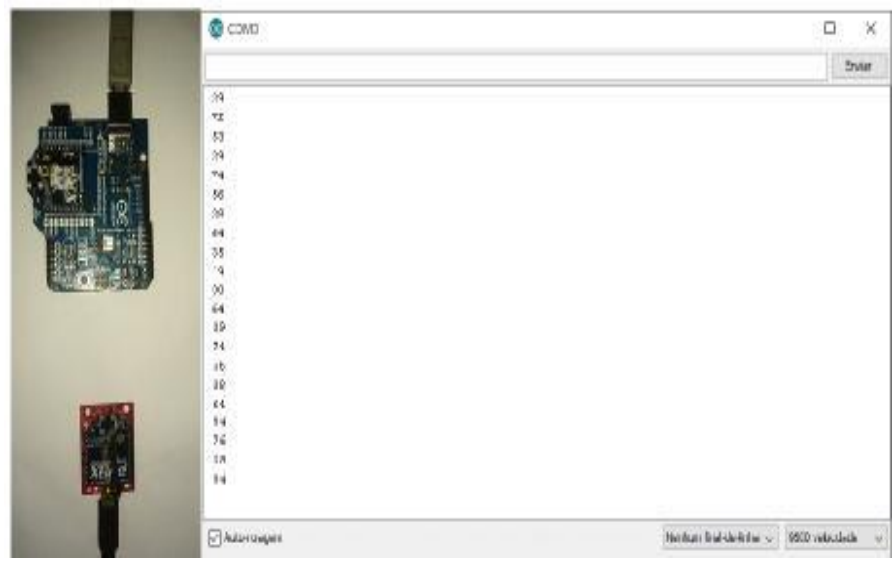

Figura 3. Distância entre os dispositivos sendo mostrada pelo Monitor Serial do Arduino.

\section{III.2 IDENTIFICAÇÃO DE OBSTÁCULOS}

Foi definida a utilização de sensores ultrassônicos com poder de alcance de até 4 metros podendo essa distância ser programada para a identificação de obstáculos em um range específico que melhor se aplique as necessidades do projeto.

Para efeito de teste sobre a eficiência do sensor foi executada no Arduino uma programação utilizando a fórmula demonstrada anteriormente para que dois ranges de distâncias fosse lidos, a primeira medida vai de $10 \mathrm{~cm}$ a $200 \mathrm{~cm}$ e acende um led verde quando existe a presença de um obstáculo e o segundo range vai de 210 a $350 \mathrm{~cm}$ acendendo um led vermelho quando feita a identificação de algum obstáculo.

A figura 4 mostra o teste de detecção de um objeto tendo sua distância mensurada na tela serial do software Arduino.

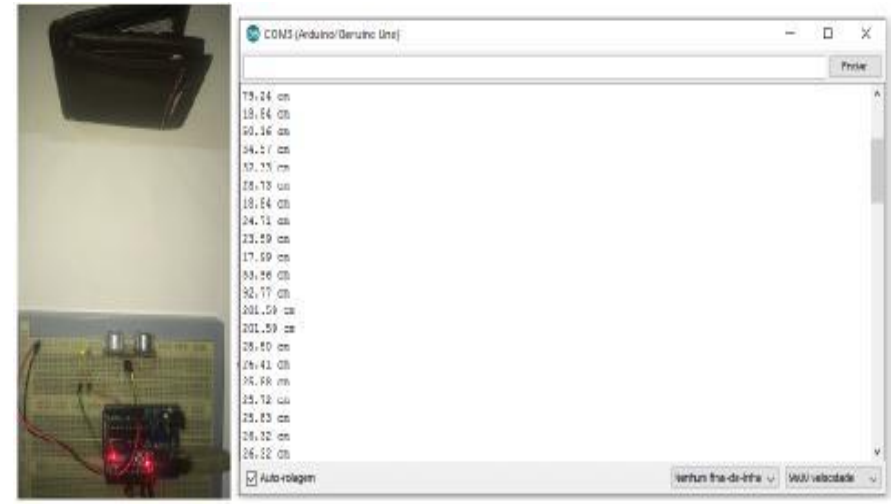

Figura 4. Detecção de obstáculos com a demonstração da distância no monitor Serial do software Arduino.

\section{III.3 MÓdULO DE ÁUDIO}

Para implementar o módulo sonoro é necessário integrá-lo ao Arduino pois a programação do microcontrolador permitiu que os arquivos em formato de áudio previamente salvos em um cartão micro $s d$ fossem executados com a utilização de um fone.

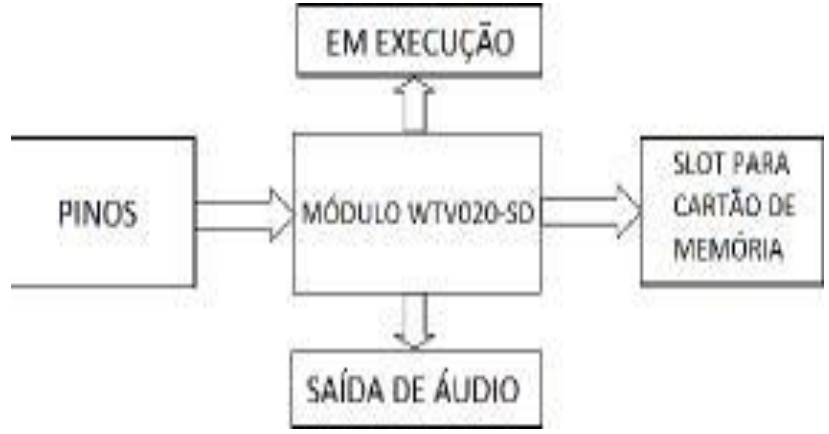

Figura 5. Representação de Aplicação do Módulo de Áudio. Fonte: [9].

\section{CONCLUSÕES}

Este artigo abordou testes iniciais relacionados à técnica de estimativa de localização, identificação de obstáculos e o módulo de áudio os resultados obtidos satisfazem as necessidades para prosseguir com o desenvolvimento do projeto.

O sistema de estimativa de localização utilizando rede ZigBee mostrou-se confiável e por permitir que a comunicação possa ser feita entre vários dispositivos numa mesma rede a precisão é maior para delimitar o posicionamento da pessoa com deficiência visual.

Para a detecção de obstáculos o sensor ultrassônico escolhido demonstrou ser capaz de atender as necessidades do projeto podendo ainda mensurar a distância do sensor até o objeto detectado.

O módulo de áudio atendeu as necessidades do projeto conseguindo enviar comandos de voz por meio de um fone, podendo ser aprimorado para futuramente indicar direções a serem tomadas pela pessoa com deficiência visual.

Podendo operar em conjunto, o sistema se torna um dispositivo compacto que pode vir a atuar de maneira ativa na vida de uma pessoa com deficiência visual podendo reduzir as dificuldades enfrentadas por elas e promover uma integração social.

\section{REFERÊNCIAS BIBLIOGRÁFICAS}

[1] INSITUTO BERASILEIRO DE GEOGRAFIA E ESTATÍSTICA (IBGE). Normas de apresentação tabular. Rio de Janeiro: Características gerais da população, religião e pessoas com deficiência, 2010.

[2] LUOMALA, J.; HAKALA, I. Towards adaptive localization in wireless sensor networks. In: IEEE. Ubiquitous Positioning, Indoor Navigation, and Location Based Service (UPINLBS), 2012. [S.1.], 2012. p. 1-8.

[3] HU-SHENG, N. et al. An improved method of self-adaptive localization for wireless sensor network in dynamic indoor environment. In: IEEE. Control Conference (CCC), 2012 31st Chinese. [S.1.], 2012. p. 6574-6577.

[4] DANTAS, M. Redes de comunicação e computadores: abordagem quantitativa. Visual Books, 2010. ISBN 9788575022559. Disponível em: <https://books.google.com.br/books?id=LbNDQwAACAAJ > 
[5] ERIC, T. Infrared vs. ultrasonic-what you should know.

Retrieved April, v. 20, p. 2012, 2008. Disponível em:

<http://www.societyofrobots.com/member tutorials/node/71>.

[6] PEREIRA, F. G. Navegação e desvio de obstáculos usando um robô móvel dotado de sensor de varredura laser. Dissertação

(Mestrado) - Universidade Federal do Espirito Santo-UFES, 2006.

[7] ELECFREAKS TECHNOLOGY LTD. Ultrasonic Ranging

Module HC - SR04. [S.1.], s.d. Disponível em:

〈http://www.micropik.com/PDF/HCSR04.pdf >.

[8] MCROBERTS, M. Arduino básico. [S.1.]: Apress Inc., 2011.

[9] ELECHOUSE ELECTRONICS TECHNOLOGY CO.

WTV020-SD MODULE. [S.1.], s.d. Disponível em:

<http://www.1 pico.com/wp-content/uploads/2015/07/WTV020-

SD.pdf $>$. 